EESTI NSV TEADUSTE AKADEEMIA TOIMETISED. IX KÖIDE

FOOSIKALIS-MATEMAATILISTE JA TEHNILISTE TEADUSTE SEERIA. 1960, NR. 3

ИЗВЕСТИЯ АКАДЕМИИ НАУК ЭСТОНСКОЙ ССР. ТОМ IХ СЕРИЯ ФИЗИКО-МАТЕМАТИЧЕСКИХ И ТЕХНИЧЕСКИХ НАУК. 1960, № 3

\title{
О НЕКОТОРЫХ ИТЕРАЦИОННЫХ МЕТОДАХ ДЛЯ РЕШЕНИЯ ОПЕРАТОРНЫХ УРАВНЕНИЙ В ПРОСТРАНСТВЕ ГИЛЬБЕРТА
}

\section{л. кивистик}

1. Рассмотрим (в общем нелинейное) уравнение

$$
P(x)=0
$$

где $P(x)$ - дважды дифференцируемый * оператор из вещественного гильбертова пространства $H$ в то же пространство. Заменим операторное уравнение (1) эквивалентным ему функциональным уравнением

$$
F(x)=0
$$

где $F(x)=\|P(x)\|^{\alpha}, \quad \frac{1}{2}<\alpha<\infty$ **.

Такую замену в случаях $\alpha=1$ и $\alpha=2$ рассмотрел Альтман [1-3]. Для решения функционального уравнения (2) (в банаховом пространстве) Альтман [6] рекомендовал итерационные методы типа

$$
x_{n+1}=x_{n}-\frac{F\left(x_{n}\right)}{F^{\prime}\left(x_{n}\right) y_{n}} y_{n} \quad(n=0,1, \ldots)
$$

где $y_{n}$ - элементы рассматриваемого пространства.

В нашем случае $F^{\prime}(x) y=\alpha\|P(x)\|^{\alpha-2}\left(P^{\prime}(x) y, P(x)\right)$ и (3) принимает вид

$$
x_{n+1}=x_{n}-\frac{\left\|P\left(x_{n}\right)\right\|^{2}}{a\left(P^{\prime}\left(x_{n}\right) y_{n}, P\left(x_{n}\right)\right)} y_{n} \quad(n=0,1, \ldots)
$$

где $y_{n} \in H$, а $x_{0}$-некоторое известное начальное приближение к решению уравнения (1). В зависимости от выбора последовательности элементов $\left\{y_{n}\right\}$ из (4) получаются разные итерационные методы. Мы рассмотрим здесь две возможности выбора элементов $y_{n}:$ 1) $y_{n}=Q\left(x_{n}\right) \equiv$ $\equiv \overline{P^{\prime}\left(x_{n}\right)} P\left(x_{n}\right)$, где $\overline{P^{\prime}(x)}$ - сопряженный к $P^{\prime}(x)$ линейный оператор, и 2) $y_{n}=P\left(x_{n}\right)$. В первом случае получим класс методов

* Дифференцируемость понимается здесь и в дальнейшем в смысле Фреше.

** Для $0<\alpha \leqslant \frac{1}{2}$ предположения последующих теорем были бы противоречивы. 


$$
x_{n+1}=x_{n}-\frac{\left\|P\left(x_{n}\right)\right\|^{2}}{\alpha \|\left. Q\left(x_{n}\right)\right|^{2}} Q\left(x_{n}\right)
$$

рассмотренный при $\alpha=1$ и $\alpha=2$ Альтманом $\left[{ }^{1-3}\right]$; во втором случае класс методов

$$
x_{n+1}=x_{n}-\frac{\left\|P\left(x_{n}\right)\right\|^{2}}{a\left(P^{\prime}\left(x_{n}\right)^{P}\left(x_{n}\right), P\left(x_{n}\right)\right)} P\left(x_{n}\right)
$$

рассмотренный при $\alpha=1$ и $\alpha=2$ в работах [1, 4, 7].

Отметим еще, что если выбрать $y_{n}=\left[P^{\prime}\left(x_{n}\right)\right]^{-1} P\left(x_{n}\right)$ и $\boldsymbol{\alpha}=1$, то получим известный метод Ньютона.

2. Выведем некоторые соотношения и оценки, существенные для исследования сходимости рассматриваемых методов.

Для всех методов (4) (в том числе и для методов (5) и (6)) справедливо соотношение

$$
\left(Q\left(x_{n}\right), x_{n+1}-x_{n}\right)=-\frac{1}{a}\left\|P\left(x_{n}\right)\right\|^{2}
$$

Так как оператор $P(x)$ дважды дифференцируем, то, учитывая (7), получим

$$
\begin{gathered}
2\left(P\left(x_{n+1}\right), P\left(x_{n}\right)\right)= \\
=2\left(1-\frac{1}{a}\right)\left\|P\left(x_{n}\right)\right\|^{2}+\left(P^{\prime \prime}\left(\bar{x}_{n}\right)\left(x_{n+1}-x_{n}\right)^{2}, P\left(x_{n}\right)\right)
\end{gathered}
$$

где $\bar{x}_{n}=x_{n}+\tau_{n}\left(x_{n+1}-x_{n}\right), 0 \leqslant \tau_{n} \leqslant 1 . \quad$ Так как

$$
\begin{gathered}
\left\|P\left(x_{n+1}\right)\right\|^{2}-2\left(P\left(x_{n+1}\right), P\left(x_{n}\right)\right)+\left\|P\left(x_{n}\right)\right\|^{2}=\left\|P\left(x_{n+1}\right)-P\left(x_{n}\right)\right\|^{2} \leqslant \\
\leqslant\left\|P^{\prime}\left(x_{n}^{\prime}\right)\left(x_{n+1}-x_{n}\right)\right\|^{2}
\end{gathered}
$$

где $x_{n}^{\prime}=x_{n}+\tau_{n}^{\prime}\left(x_{n+1}-x_{n}\right), \quad 0 \leqslant \tau_{n}^{\prime} \leqslant 1$, то в силу (4) и (8) получим оценку

$$
\begin{array}{r}
\left\|P\left(x_{n+1}\right)\right\|^{2} \leqslant\left[1-\frac{2}{a}+\frac{1}{a^{2}}\left(\left\|P^{\prime}\left(x_{n}^{\prime}\right)\right\|^{2}+\right.\right. \\
\left.\left.+\left\|P^{\prime \prime}\left(\overline{x_{n}}\right)\right\|\left\|P\left(x_{n}\right)\right\|\right) \frac{\left\|P\left(x_{n}\right)\right\|^{2}\left\|y_{n}\right\|^{2}}{\left(y_{n}, Q\left(x_{n}\right)\right)^{2}}\right]\left\|P\left(x_{n}\right)\right\|^{2}
\end{array}
$$

Учитывая, что оператор $Q(x)=\overline{P^{\prime}(x)} P(x)$ дифференцируем, мож. но получить и другую оценку. Формула Тейлора для функционала $\|P(x)\|^{2}$ с остаточным членом в форме Лагранжа дает

$$
\begin{gathered}
\left\|P\left(x_{n+1}\right)\right\|^{2}=\left\|P\left(x_{n}\right)\right\|^{2}+2\left(Q\left(x_{n}\right), x_{n+1}-x_{n}\right)+ \\
+\left(Q^{\prime}\left(x_{n}^{\prime \prime}\right)\left(x_{n+1}-x_{n}\right), \quad x_{n+1}-x_{n}\right)
\end{gathered}
$$

где $x_{n}^{\prime \prime}=x_{n}+\tau_{n}^{\prime \prime}\left(x_{n+1}-x_{n}\right), 0 \leqslant \tau^{\prime \prime}{ }_{n} \leqslant 1$. Учитывая (7) и (4), получим

$$
\left\|P\left(x_{n+1}\right)\right\|^{2} \leqslant\left(1-\frac{2}{a}+\frac{1}{a^{2}} \frac{\left\|Q^{\prime}\left(x_{n}^{\prime \prime}\right)\right\|\left\|P\left(x_{n}\right)\right\|^{2}\left\|y_{n}\right\|^{2}}{\left(y_{n}, Q\left(x_{n}\right)\right)^{2}}\right)\left\|P\left(x_{n}\right)\right\|^{2}
$$


3. Докажем о сходимости методов (5) следующую теорему:

Т еорем а 1. Пусть выполнены условия:

$1^{\circ} \quad\left\|P\left(x_{0}\right)\right\| \leqslant \delta_{0}$;

$2^{\circ}$ для всех $x \in S\left(x_{0}, r\right)^{*}$, где $r=\frac{M \delta_{0}}{a(1-q)}$, имеют место оценки: a) $\left\|\overline{P^{\prime}(x)} h\right\| \geqslant \frac{1}{M}\|h\|$ для всех $h \in H(M>0)$,

б) $\left\|P^{\prime}(x)\right\| \leqslant A, \quad$ в) $\left\|P^{\prime \prime}(x)\right\| \leqslant B$;

$3^{\circ} \quad q=\frac{1}{a} \sqrt{\alpha^{2}-2 \alpha+M^{2}\left(A^{2}+B \delta_{0}\right)}<1$.

Тогда уравнение (1) имеет в сфере $S\left(x_{0}, r\right)$ решение $x^{*}, \kappa$ которому сходится последовательность $\left\{x_{n}\right\}$, полученная методом (5), и справедлива оценка

$$
\left\|x^{*}-x_{n}\right\| \leqslant \frac{M}{a(1-q)}\left\|P\left(x_{n}\right)\right\| \leqslant \frac{M \delta_{0}}{a(1-q)} \cdot q^{n}
$$

Д ок аз ательств о. Покажем, что если $x_{0}$ заменить на $x_{1}$, то все условия $1^{\circ}-3^{\circ}$ по-прежнему выполнены. Учитывая условия $2^{\circ}$ a) и $1^{\circ}$, получим из (5)

$$
\left\|x_{1}-x_{0}\right\| \leqslant \frac{M}{a}\left\|P\left(x_{0}\right)\right\|<r
$$

т. е. $x_{1} \in S\left(x_{0}, r\right)$. Поэтому, используя условия $2^{\circ}$ и учитывая, что $y_{n}=$ $=Q\left(x_{n}\right)$, получим из $(9)$

$$
\left\|P\left(x_{1}\right)\right\|^{2} \leqslant \frac{1}{\alpha^{2}}\left[\alpha^{2}-2 \alpha+M^{2}\left(A^{2}+B \delta_{0}\right)\right]\left\|P\left(x_{0}\right)\right\|^{2}
$$

Это значит, что найдется постоянная $\delta_{1}$, которая удовлетворяет условию $1^{\circ}$ при $x_{1}$ :

$$
\left\|P\left(x_{1}\right)\right\| \leqslant \delta_{1} \leqslant q\left\|P\left(x_{0}\right)\right\| \leqslant q \delta_{0}<\delta_{0}
$$

Условия $2^{\circ}$ выполнены также для точки $x_{1}$, так как $S\left(x_{1}, r_{1}\right) \subset S\left(x_{0}, r\right)$, где $r_{1}=\frac{M \delta_{1}}{a\left(1-q_{1}\right)}, q_{1}=\frac{1}{a} \sqrt{\alpha^{2}-2 \alpha+M^{2}\left(A^{2}+B \delta_{1}\right)}<q<1$. Докажем, что $S\left(x_{1}, r_{1}\right) \subset S\left(x_{0}, r\right)$. Пусть $x \in S\left(x_{1}, r_{1}\right)$; тогда $\left\|x-x_{0}\right\| \leqslant \| x-$ $-x_{1}\|+\| x_{1}-x_{0} \| \leqslant \frac{M \delta_{1}}{a\left(1-q_{1}\right)}+\frac{M \delta_{0}}{a} \leqslant \frac{M \delta_{0}}{a(1-q)}=r$, т. е. $x \in S\left(x_{0}, r\right)$ и, таким образом, $S\left(x_{1}, r_{1}\right) \subset S\left(x_{0}, r\right)$.

Итак, для $x_{1}$ выполнены условия $1^{\circ}-3^{\circ}$ с заменой чисел $\delta_{0}, r, q$ на $\delta_{1}, r_{1}, q_{1}$. Это позволяет продолжать последовательное определение элементов $x_{n}$. Вместе с тем получим при всех $n$

$$
\left\|P\left(x_{n+1}\right)\right\| \leqslant \delta_{n+1} \leqslant q_{n}\left\|P\left(x_{n}\right)\right\| \leqslant q^{n+1} \delta_{0}
$$

где $q_{n}=\frac{1}{a} \sqrt{\alpha^{2}-2 \alpha+M^{2}\left(A^{2}+B \delta_{n}\right)}<q_{n-1} \leqslant q<1 \quad\left(q_{0}=q\right)$;

- Символом $S\left(x_{0}, r\right)$ обозначена сфера $\left\|x-x_{0}\right\| \leqslant r$. 


$$
x_{n+1} \in S\left(x_{n}, r_{n}\right) \subset S\left(x_{0}, r\right)
$$

где $r_{n}=\frac{M \delta_{n}}{a\left(1-q_{n}\right)}$

$$
\left\|x_{n+1}-x_{n}\right\| \leqslant \frac{M}{a}\left\|P\left(x_{n}\right)\right\|
$$

Поэтому в силу (14) и (12)

$$
\begin{gathered}
\left\|x_{n+p}-x_{n}\right\| \leqslant\left\|x_{n+p}-x_{n+p-1}\right\|+\ldots+\left\|x_{n+2}-x_{n+1}\right\|+\left\|x_{n+1}-x_{n}\right\| \leqslant \\
\leqslant \frac{M}{a}\left(\left\|P\left(x_{n+p-1}\right)\right\|+\ldots+\left\|P\left(x_{n+1}\right)\right\|+\left\|P\left(x_{n}\right)\right\|\right) \leqslant \frac{M}{a}\left\|P\left(x_{n}\right)\right\|\left(q_{n}^{p-1}+\ldots\right. \\
\left.\ldots+q_{n}+1\right)=\frac{M\left(1-q_{n}^{p}\right)}{a\left(1-q_{n}\right)}\left\|P\left(x_{n}\right)\right\|<\frac{M}{a(1-q)}\left\|P\left(x_{n}\right)\right\| \leqslant \frac{M \delta_{0}}{a(1-q)} q^{n} .
\end{gathered}
$$

Это доказывает существование предела $\lim _{n \rightarrow \infty} x_{n}=x^{*} \in H$. Переходя к пределу при $p \rightarrow \infty$, получим (11), откуда одновременно видно, что $x^{*} \in S\left(x_{0}, r\right)$. Так как оператор $P(x)$ непрерывен в $S\left(x_{0}, r\right)$, то $\left\|P\left(x^{*}\right)\right\|=\lim \left\|P\left(x_{n}\right)\right\| \leqslant \delta_{0} \lim q^{n}=0$, или $P\left(x^{*}\right)=0$, т. е. $x^{*}$ является решением уравнения (1).

Теорема доказана.

4. Если учитывать вместо (9) оценку (10), то мсжно аналогичным образом доказать следующую теорему:

Т еорем а 1а. Пусть выполнены условия:

$1^{\circ} \quad\left\|P\left(x_{0}\right)\right\| \leqslant \delta_{0}$

$2^{\circ}$ для всех $x \in S\left(x_{0}, r\right)$, где $r=\frac{M \delta_{0}}{a(1-q)}$, имеют место оценки:

а) $\left\|\overline{P^{\prime}(x)} h\right\| \geqslant \frac{1}{M}\|h\|$ для всех $h \in H(M>0)$,

б) $\left\|Q^{\prime}(x)\right\| \leqslant K$;

$3^{\circ} \quad q=\frac{1}{c} \sqrt{\alpha^{2}-2 \alpha+M^{2} K}<1$.

Тогда имеют место утверждения теоремь 1.

Примечание 1. Если в теоремах 1 и 1 а условие $2{ }^{\circ}$ a) заменить более строгим условием

$$
\left|\left(P^{\prime}(x) h, h\right)\right| \geqslant \frac{1}{M}\|h\|^{2} \text { для всех } h \in H \quad(M>0)
$$

(не изменяя других условий), то утверждения теорем остаются в силе, но оценку (11) можно заменить более точной:

$$
\left\|x^{*}-x_{n}\right\| \leqslant M\left\|P\left(x_{n}\right)\right\| \leqslant M \delta_{0} q^{n}
$$

Действительно, из (15) вытекает $2^{\circ}$ a). С другой стороны, учитывая (13), (15) и то, что $x^{*} \in S\left(x_{0}, r\right)$, получим 


$$
\begin{gathered}
\left\|P\left(x_{n}\right)\right\|\left\|x^{*}-x_{n}\right\| \geqslant\left|\left(P\left(x^{*}\right)-P\left(x_{n}\right), x^{*}-x_{n}\right)\right|= \\
=\left|\left(P^{\prime}(\bar{x})\left(x^{*}-x_{n}\right), x^{*}-x_{n}\right)\right| \geqslant \frac{1}{M}\left\|x^{*}-x_{n}\right\|^{2}
\end{gathered}
$$

где $\bar{x}=x_{n} \div \tau\left(x^{*}-x_{n}\right), \quad 0 \leqslant \tau \leqslant 1$. Из последних неравенств следует (16)

5. Учитывая примечание 1 , можно аналогично доказательству теоремы 1 доказать следующие теоремы о сходимости методов (6) (которые справедливы и для методов (5)):

Т ео рем а 2. Пусть выполнены условия теоремы 1, за исключением $2^{\circ}$ а), которое заменено условием (15). Тогда уравнение (1) имеет в сфере $\left\|x-x_{0}\right\| \leqslant M\left\|P\left(x_{0}\right)\right\|$ решение $x^{*}$, $\kappa$ которому сходится последовательность $\left\{x_{n}\right\}$, полученная методом (6) (или методом (5)), и справедлива оценка (16).

Т ео рем а 2а. Пусть выполнены условия теоремы 1а, за исключе нием $\left.2^{\circ} \mathrm{a}\right)$, которое заменено условием (15). Тогда имеют место утвер. ждения теоремы 2.

Пр и ме чани е 2. В условиях теорем 2 и 2а уравнение (1) имеет в сфере $S\left(x_{0}, r\right)$ единственное решение. Это непосредственное следствие из теоремы 8 статьи [7].

6. Легко проверить, что из условий $2^{\circ *}$ теорем 1 и 2 следует неравенство $M^{2}\left(A^{2}+B \delta_{0}\right) \geqslant 1$.

Пусть $x^{*}$ - решение уравнения (1), находящееся в сфере $S\left(x_{0}, r\right)$. Тогда имеет место равенство

$$
Q^{\prime}\left(x^{*}\right)=\overline{P^{\prime}\left(x^{*}\right)} P^{\prime}\left(x^{*}\right)
$$

Так как $x^{*} \in S\left(x_{0}, r\right)$, то в силу (17) и условия $2^{\circ}$ теорем $1 \mathrm{a}, 2 \mathrm{a}\left\|P^{\prime}\left(x^{*}\right)\right\|^{2}=$ $=\left\|Q^{\prime}\left(x^{*}\right)\right\| \leqslant K$ и $M^{2} K \geqslant 1$.

Условие $3^{\circ}$ теорем $1-2$ а равносильно условию

где

$$
c<2 \alpha,
$$

$$
1 \leqslant c= \begin{cases}M^{2}\left(A^{2}+B \delta_{0}\right) & \text { для теорем } 1 \text { и } 2 \\ M^{2} K & \text { для теорем 1а и } 2 \mathrm{a}\end{cases}
$$

Радиус $r$ сферы $S\left(x_{0}, r\right)$ в условии $2^{\circ}$ этих теорем является функцией от $\alpha$ :

$$
r=r(\alpha)=\frac{M \delta_{0}}{a(1-q)}=\frac{M \delta_{0}}{a-\sqrt{a^{2}-2 a+c}}=\frac{a+\sqrt{a^{2}-2 a+c}}{2 a-c} M \delta_{0}
$$

которая уменьшается с возрастанием $\alpha$ (если $c>1)$ и значения которой становятся сколь угодно близкими к $M \delta_{0}$, если только $\alpha$ становится достаточно большой по сравнению с $c$ (если $c=1$, то при $\alpha \geqslant 1$

* Условиями $2^{\circ}$ теорем 2 и 2 а считаем соответственно условия $2^{\circ}$ теорем 1 и 1 а, где $2^{\circ}$ a) заменено условием (15); условия $1^{\circ}$ и $3^{\circ}$ этих теорем - это соответственно условия $1^{\circ}$ и $3^{\circ}$ теорем 1 и 1 а. 
$\left.r=M \delta_{0}\right)$. Следовательно, подходящим выбором $\alpha$ всегда можно достичь выполнения неравенств (18) и $r(\alpha) \leqslant R$, где $R>M \delta_{0}$. Итак, справедлива следующая теорема:

Т еорем а 3. Если существуют такие постоянные $M, A$ и $B$ (соответственно $M$ и K), что все неравенства в условии $2^{\circ}$ теорем 1 и 2 (соот. ветственно теорем 1а и 2а) выполнены для всех х из некоторой сферы $S\left(x_{0}, R\right)$, где $R>M \delta_{0}$, то всегда найдется вещественное число $\bar{\alpha}$, при котором имеют место утверждения теорем 1 и 2 (соответственно 1а u 2а), если только в формулах (5) $u$ (6) положить $\alpha \geqslant \bar{\alpha}$.

П ри м е ч ан и е 3. Априорная оценка погрешности в теоремах $1-2$ a обеспечивает сходимость методов (5) и (6) с быстротой геометрической прогрессии. При этом минимальным значением знаменателя прогрессии для данной $c$ является

$$
q_{\min }=\sqrt{1-\frac{1}{c}}
$$

которое достигается при $\alpha=c$.

Примечани е 4. В предыдущих теоремах желательнее пользоваться апостериорными оценками. Но так как вычисление $\left\|P\left(x_{n}\right)\right\|$ требует лишней работы, а $\left\|P\left(x_{n}\right)\right\| \leqslant q\left\|P\left(x_{n-1}\right)\right\|$, причем $\left\|P\left(x_{n-1}\right)\right\|$ уже вычислена при нахождении $x_{n}$, то можно пользоваться и (менее точными) оценками

$$
\begin{aligned}
& \left\|x^{*}-x_{n}\right\| \leqslant \frac{M q}{a(1-q)}\left\|P\left(x_{n-1}\right)\right\| \text { в теоремах } 1,1 \text { a } \\
& \left\|x^{*}-x_{n}\right\| \leqslant M q\left\|P\left(x_{n-1}\right)\right\| \quad \text { в теоремах } 2,2 \mathrm{a}
\end{aligned}
$$

При меч ани е 5 . Если условие $3^{\circ}$ теорем $1-2$ а заменить более строгим условием, то оченки $\left.2^{\circ} \mathrm{a}\right), 6$ ), в) можно находить в меньшей области, чем в теоремах $1-2 \mathrm{a}$. Теореме 2 , например, соответствует тогда следующая теорема:

Т еорем а $22_{1}$. Пусть выполнены условия:

$1^{\circ} \quad\left\|x_{1}-x_{0}\right\|=\frac{\left\|P\left(x_{0}\right)\right\|^{3}}{a\left|\left(P^{\prime}\left(x_{0}\right) P\left(x_{0}\right), P\left(x_{0}\right)\right)\right|} \leqslant \eta_{0} ;$

$2^{\circ}$ для всех $x \in S\left(x_{0}, r\right)$, где $r=\frac{\eta_{0}}{1-q}$, имеют место оценки:

а) $\left|\left(P^{\prime}(x) h, h\right)\right| \geqslant \frac{1}{M}\|h\|^{2}$ для всех $h \in H(M>0)$,

б) $\left\|P^{\prime}(x)\right\| \leqslant A, \quad$ в) $\left\|P^{\prime \prime}(x)\right\| \leqslant B$;

3. $q<1$, где $q= \begin{cases}\frac{1}{a} \sqrt{\alpha^{2}-2 \alpha+M^{2}\left(A^{2}+A B \alpha \eta_{0}\right)}, & \text { если } \alpha \leqslant 2, \\ \frac{1}{a} \sqrt{(\alpha-1)^{2} M^{2} A^{2}+M^{2} A B \alpha \eta_{0}}, & \text { если } \alpha>2 .\end{cases}$

Тогда уравнение (1) имеет в сфере $\left\|x-x_{0}\right\| \leqslant M\left\|P\left(x_{0}\right)\right\|$ решение $x^{*}$, $\kappa$ которому сходится последовательность $\left\{x_{n}\right\}$, полученная методом (5) или методом (6), и справедлива оценка 


$$
\left\|x^{*}-x_{n}\right\| \leqslant M\left\|P\left(x_{n}\right)\right\| \leqslant \alpha \eta_{0} q^{n}
$$

Если $\alpha \leqslant 2$, то теорема $2_{1}$ может дать лучшие результаты, чем теорема 2 . Но если $\alpha>2$, то условие $3^{\circ}$ теоремы $2_{1}$ значительно строже условия $3^{\circ}$ теоремы 2. В самом деле, если условие $3^{\circ}$ теоремы 2 равносильно требованию $M^{2}\left(A^{2}+B \delta_{0}\right)<2 \alpha$, то условие $3^{\circ}$ теоремы $2_{1}$ в случае $\alpha>2$ требует, чтобы было $M^{2}\left(A^{2}+B \delta_{0}\right) \leqslant M^{2}\left(A^{2}+A B \alpha \eta_{0}\right)<\left(\frac{a}{a-1}\right)^{2}<4$. Это значит, что в данном случае теорема 3 недействительна.

Теорему $2_{1}$ можно доказать аналогично теоремам $1-2 \mathrm{a}$, нужно только иметь в виду, что теперь справедливо неравенство $\left\|x_{n+2}-x_{n+1}\right\| \leqslant$ $\leqslant q\left\|x_{n+1}-x_{n}\right\|(n=0,1, \ldots)$.

В частном случае $\alpha=2$ эта теорема совпадает с результатом Альтмана о сходимости метода (6) (см. [ $\left.{ }^{4}\right]$, теорема 2). В приведенной здесь теореме уточнена оценка погрешности и имеются другие несущественные различия.

Можно доказать и теоремы, соответствующие теоремам 1, 1a, 2a (в том же самом смысле, как теорема $22_{1}$ соответствует теореме 2) и дающие в частном случае $\alpha=2$ результаты, полученные Альтманом $\left[{ }^{3,4}\right.$ ] для методов (5) и (6) (где $\alpha=2$ ). Мы этих теорем не приводим, так как в случае $\alpha>2$ они страдают тем же недостатком, что и теорема $2_{1}$.

7. Приме ч а и и е 6 . Как уже отмечено (см. [ $\left.{ }^{7}\right]$, стр. 145 , подстрочное примечание), предположения теорем 2 и 4 статьи ['] противоречивы. Из сказанного в начале пункта 6 следует, что противоречивы и условия теорем 1 и 3 той же статьи. Вместе с тем из теорем $1-2$ а и 21 данной статьи видно, что для устранения противоречий можно в [1] условие $1^{\circ}$ теоремы 1 заменить условием $B^{2} K<2$ и условие $5^{\circ}$ теоремы 2 условием $B^{2}\left(E^{2}+K D\right)<2$, изменяя соответственно и постоянные $r$ и $\alpha$ в этих теоремах.

При меч ан и е 7 . На основе теоремы 2 -статьи $\left[{ }^{2}\right]$ и на основе статьи [5] можно предположить, что при $\alpha=2$ для методов (5) и (6) найдены эффективные условия, при которых сходимость этих методов второго порядка. Но оказывается, что это не так.

\section{Докажем это.}

Допустим, что условие $3^{\circ}$ теоремы 2 в [2] выполнено, т. е. $2 h_{0} \leqslant 1$. Тогда из доказательства теоремы 1 той же статьи $\left.{ }^{2}\right]$ следует (так как теорема 2 - следствие из теоремы 1 ), что $2 h_{n} \leqslant 2 h_{0} \leqslant 1$; значит и $\lim 2 h_{n} \leqslant 1$. Так как $x^{*} \in S\left(x_{0}, r\right)$, то из условия $2^{\circ}$

$$
n \rightarrow \infty
$$

теоремы 2 в ${ }^{[2]}$ и из равенства (17) настоящей работы получим $K \geqslant\left\|Q^{\prime}\left(x^{*}\right)\right\|=\left\|P^{\prime}\left(x^{*}\right)\right\|^{2}$. Но это значит, что $\lim 2 h_{n} \geqslant \lim \frac{K\left\|P\left(x_{n}\right)\right\|^{2}}{\left\|Q\left(x_{n}\right)\right\|^{2}} \geqslant \lim \frac{K}{\left\|P^{\prime}\left(x_{n}\right)\right\|^{2}} \geqslant 1$.

Таким образом, в случае $2 h_{0}<1$ мы получили противоречие, и возможен только случай $2 h_{0}=1$. Но тогда оценка погрешности этой теоремы не обеспечивает сходимости второго порядка.

Условия обеих теорем статьи[5] тоже противоречивы. В самом деле, если предполагать, что условне (5) этой статьи $\left(h_{0}=B_{0} K \eta_{0} \leqslant \frac{1}{2}\right)$ выполнено, то из доказательства теоремы 1 в [5] следует, что $h_{n} \leqslant h_{0} \leqslant \frac{1}{2}$. Но в силу условий той же теоремы $h_{n}=$ $=B_{n} K \eta_{n} \geqslant \frac{K\left\|P\left(x_{n}\right)\right\|^{3}}{2\left(P^{\prime}\left(x_{n}\right) P\left(x_{n}\right), P\left(x_{n}\right)\right)^{2}} \geqslant \frac{K}{\left.2\left\|P^{\prime}\left(x_{n}\right)\right\|\right|^{2}\left\|P\left(x_{n}\right)\right\|} \rightarrow \infty$ при $n \rightarrow \infty$, что противоречит требованию (5) из [5]. Отметим, что неравенство (6) этой теоремы также не имеет места, но можно обойтись и без этого требования. 
На одно противоречие теоремы 2 статьи [5] обратил внимание Вайнберг (см. РЖМат. 1958, 7929, примечание референта). Сверх того противоречиво и условие (5), выполнение которого требуется в той же теореме.

8. Если условие $2^{\circ}$ a) теорем $1-2$ а заменить (легче проверяемым) условием

$$
\left\|\overline{P^{\prime}\left(x_{0}\right)} h\right\| \geqslant \frac{1}{M_{0}}\|h\| \text { для всех } h \in H \quad\left(M_{0}>0\right)
$$

\section{нли соответственно}

$$
\left|\left(P^{\prime}\left(x_{0}\right) h, h\right)\right| \geqslant \frac{1}{M_{0}}\|h\|^{2} \text { для всех } h \in H\left(M_{0}>0\right)
$$

то имеют место следующие теоремы, доказательство которых можно провести аналогично доказательству теоремы 3 в [ [7]:

Теорем а 4. Пусть выполнены условия:

$1^{\circ}\left\|P\left(x_{0}\right)\right\|=\delta_{0} \leqslant \bar{\delta}_{0}$;

$2^{\circ}\left\|\overline{P^{\prime}\left(x_{0}\right)} h\right\| \geqslant \frac{1}{M_{0}}\|h\|$ для всех $h \in H\left(M_{0}>0\right) ;$

$3^{\circ}$ для всех $x \in S\left(x_{0}, r\right)$, где $r=\frac{1}{B}\left(\frac{1}{M_{0}}-\frac{1}{M^{*}}\right) \frac{\delta_{0}}{\bar{\delta}_{0}}$, имеют место оценки

$$
\left\|P^{\prime}(x)\right\| \leqslant A,\left\|P^{\prime \prime}(x)\right\| \leqslant B ;
$$

$4^{\circ}$ величины $M_{0}, \bar{\delta}_{0}, A$ и В такие, что последовательности $\left\{M_{n}\right\}$ и $\left\{\bar{\delta}_{n}\right\}$, вычисленные из рекуррентных соотношений

$$
\begin{gathered}
M_{n+1}=\frac{M_{n}}{1-\frac{1}{a} M_{n}^{2} B \bar{\delta}_{n}}, \\
\bar{\delta}_{n+1}=\bar{\delta}_{n} \cdot \frac{1}{\alpha} \sqrt{\alpha^{2}-2 \alpha+M_{n}^{2}\left(A^{2}+B \bar{\delta}_{n}\right)},
\end{gathered}
$$

сходятся (так что $\frac{1}{a} M_{n}^{2} B \bar{\delta}_{n}<1$ для всех $\left.n\right)$.

Тогда уравнение (1) имеет в сфере $S\left(x_{0}, r\right)$ решение $x^{*}, \kappa$ которому сходится последовательность $\left\{x_{n}\right\}$, полученная методом (5), и справедлива оценка

$$
\left\|x^{*}-x_{n}\right\| \leqslant \frac{1}{B}\left(\frac{1}{M_{n}}-\frac{1}{M^{*}}\right) \frac{\delta_{n}}{\bar{\delta}_{n}} \leqslant \frac{1}{B}\left(\frac{1}{M_{n}}-\frac{A}{\sqrt{2 \alpha}}\right) \frac{\delta_{n}}{\bar{\delta}_{n}},
$$

где $M^{*}=\lim _{n \rightarrow \infty} M_{n} u \delta_{n}=\left\|P\left(x_{n}\right)\right\|$.

Т е о рем а 4а. Пусть выполнены условия:

$1^{\circ}\left\|P\left(x_{0}\right)\right\|=\delta_{0} \leqslant \bar{\delta}_{0}$;

$2^{\circ}\left\|\overline{P^{\prime}\left(x_{0}\right)} h\right\| \geqslant \frac{1}{M_{0}} \quad\|h\|$ для всех $h \in H \quad\left(M_{0}>0\right) ;$ 
$3^{\circ}$ для всех $x \in S\left(x_{0}, r\right)$, где $r=\frac{1}{B}\left(\frac{1}{M_{0}}-\frac{1}{M^{*}}\right) \frac{\delta_{0}}{\bar{\delta}_{0}}$, имеют место оценки $\left\|P^{\prime \prime}(x)\right\| \leqslant B,\left\|Q^{\prime}(x)\right\| \leqslant K ;$

$4^{\circ}$ величины $M_{0}, \bar{\delta}_{0}, B$ и $К$ такие, что последовательности $\left\{M_{n}\right\}$ и $\left\{\bar{\delta}_{n}\right\}$, вынисленные из рекуррентных соотночений

$$
\begin{gathered}
M_{n+1}=\frac{M_{n}}{1-\frac{1}{a} M_{n}^{2} B \bar{\delta}_{n}}, \\
\bar{\delta}_{n+1}=\bar{\delta}_{n} \frac{1}{a} \sqrt{\alpha^{2}-2 \alpha+M_{n}^{2} K},
\end{gathered}
$$

сходлтся (так что $\frac{1}{a} M_{n}^{2} B \bar{\delta}_{n}<1$ для всех $n$ ).

Тогда уравнение (1) имеет в сфере $S\left(x_{0}, r\right)$ решение $x^{*}, \kappa$ которому сходится последовательность $\left\{x_{n}\right\}$, полученная методом (5), и справедлива оценка

$$
\text { - }\left\|x^{*}-x_{n}\right\| \leqslant \frac{1}{B}\left(\frac{1}{M_{n}}-\frac{1}{M^{*}}\right) \frac{\delta_{n}}{\bar{\delta}_{n}} \leqslant \frac{1}{B}\left(\frac{1}{M_{n}}-\sqrt{\frac{K}{2 c}}\right) \frac{\delta_{n}}{\bar{\delta}_{n}},
$$

где $M^{*}=\lim M_{n}$ и $\delta_{n}=\left\|P\left(x_{n}\right)\right\|$.

Пр и ме ч ан и е 8 . Условие $2^{\circ}$ теорем 4 и 4 а обеспечивает существование правого обратного оператора по отношению к $P^{\prime}\left(x_{0}\right)$. Пусть кроме того,

$$
\left\|P^{\prime}\left(x_{0}\right) h\right\| \geqslant \frac{1}{M_{0}}\|h\| \text { для всех } h \in H
$$

Тогда существует и левый обратный оператор и имеет место оценка $\left\|\left[P^{\prime}\left(x_{0}\right)\right]^{-1}\right\| \leqslant M_{0}$. Те же неравенства остаются в силе для $x_{n}$, если $M_{0}$ заменить на $M_{n}$.

Теперь можно в теоремах 4 и 4 a формулы для оценки погрешностей заменить более удобной формулой

$$
\left\|x^{*}-x_{n}\right\| \leqslant \frac{2 M_{n} \delta_{n}}{1+\sqrt{1-2 M_{n}^{2} B \delta_{n}^{*}}}<2 M_{n} \delta_{n}
$$

В самом деле, используя формулу Тейлора

$$
\left(P\left(x^{*}\right), h\right)=\left(P\left(x_{n}\right)+P^{\prime}\left(x_{n}\right)\left(x^{*}-x_{n}\right)+\frac{1}{2} P^{\prime \prime}\left(\bar{x}_{n}\right)\left(x^{*}-x_{n}\right)^{2}, h\right)
$$

в случае $h=\overline{\left[P^{\prime}\left(x_{n}\right)\right]^{-1}}\left(x^{*}-x_{n}\right)$, получим

$$
\frac{1}{2} M_{n} B\left\|x^{*}-x_{n}\right\|^{2}-\left\|x^{*}-x_{n}\right\|+M_{n} \delta_{n} \geqslant 0
$$

откуда и следует $(21)$ (если только $\left.2 M_{n}^{2} B \delta_{n}<1\right)$.

Т е о рем а 5. Пусть выполнены условия теоремы 4, за исключением $2^{\circ}$, которое заменено условием (20). Тогда уравнение (1) имеет в сфере $\left\|x-x_{0}\right\| \leqslant M^{*} \delta_{0}$ решение $x^{*}, \kappa$ которому сходится последовательность 
$\left\{x_{n}\right\}$, полученная методом (5) или методом (6), и имеет место оценка

$$
\left\|x^{*}-x_{n}\right\| \leqslant M^{*} \delta_{n} \leqslant \frac{\sqrt{2 a}}{A} \delta_{n}
$$

где $M^{*}=\lim M_{n} u \delta_{n}=\left\|P\left(x_{n}\right)\right\|$.

Т е ор ем а 5а. Пусть выполнены условия теоремы 4 a, за исключением $2^{\circ}$, которое заменено условием (20). Тогда уравнение (1) имеет в сфере $\left\|x-x_{0}\right\| \leqslant M^{*} \delta_{0}$ решение $x^{*}, \kappa$ которому сходится последовательность $\left\{x_{n}\right\}$, полученная методом (5) или методом (6), и имеет место оценка

$$
\left\|x *-x_{n}\right\| \leqslant M^{*} \delta_{n} \leqslant \sqrt{\frac{2 a}{K}} \delta_{n}
$$

где $M^{*}=\lim M_{n} u \delta_{n}=\left\|P\left(x_{n}\right)\right\|$.

9. Рассмотрим теперь еще более общие методы

$$
x_{n+1}=x_{n}-\frac{\left\|P\left(x_{n}\right)\right\|^{2}}{\alpha_{n}\left\|Q\left(x_{n}\right)\right\|^{2}} Q\left(x_{n}\right)
$$

и

$$
x_{n+1}=x_{n}-\frac{\left\|P\left(x_{n}\right)\right\|^{2}}{a_{n}\left(P^{\prime}\left(x_{n}\right) P\left(x_{n}\right), P\left(x_{n}\right)\right)} P\left(x_{n}\right),
$$

где $\frac{1}{2}<\alpha_{n}<\infty$. Пусть

$$
\begin{aligned}
& \alpha=\inf _{n} \alpha_{n} \\
& \beta=\sup _{n} \alpha_{n}<\infty
\end{aligned}
$$

и

$$
q=\max \left\{\frac{1}{\alpha} \sqrt{\alpha^{2}-2 \alpha+c}, \quad \frac{1}{\beta} \sqrt{\beta^{2}-2 \beta+c}\right\}
$$

Если, в частности, $\alpha_{n} \leqslant c$ для всех $n$, то $q=\frac{1}{c} \sqrt{\alpha^{2}-2 \alpha+c}$, но если $\alpha_{n} \geqslant c(n=0,1, \ldots)$, то $q=\frac{1}{\beta} \sqrt{\beta^{2}-2 \beta+c}$.

Легко убедиться, что теоремы $1-2$ а остаются в силе, если методы (5) и (6) заменить в них соответственно методами (5a) и (6a), а постоянные $\alpha$ и $q$ определить формулами (22) и (23), где $c$ определено формулой (19) .

10. Выберем в формулах (5a) и (6a) числа $\alpha_{n}$ так, что для всех $n$

$$
\begin{array}{r}
M_{n}^{2} B \bar{\delta}_{n}<\alpha_{n} \\
M_{n}^{2} A^{2}+M_{n}^{2} B \bar{\delta}_{n}<2 \alpha_{n}
\end{array}
$$

где $M_{n}, \bar{\delta}_{n}$ вычислены из рекуррентных соотношений 


$$
\begin{gathered}
M_{k+1}=\frac{M_{k}}{1-\frac{1}{\alpha_{k}} M_{k}^{2} B \bar{\delta}_{k}} \\
\bar{\delta}_{k+1}=\bar{\delta}_{k} \cdot \frac{1}{\alpha_{k}} \sqrt{\alpha_{k}^{2}-2 \alpha_{k}+M_{k}^{2}\left(A^{2}+B \bar{\delta}_{k}\right)}
\end{gathered}
$$

Если окажется возможным конструировать последовательность $\left\{\alpha_{n}\right\}$ так, что $\sup _{n} \alpha_{n}<+\infty$, то последовательности $\left\{M_{n}\right\}$ и $\left\{\bar{\delta}_{n}\right\}$ сходятся и имеет место теорема, аналогичная теореме 5 :

Те орем а 6. Если выполнены условия $1^{\circ}, 3^{\circ}$ теоремы 4, условие (20) и существует такая последовательность вещественных чисел $\left\{\alpha_{n}\right\}$, что $\sup _{n} \alpha_{n}=\beta<\infty$ и имеют место неравенства (24), где $M_{n} и \bar{\delta}_{n}$ вычислены из рекуррентных соотношений (25), то уравнение (1) имеет в сфере $\left\|x-x_{0}\right\| \leqslant M^{*} \delta_{0}$ решение $x^{*}, \kappa$ которому сходится последовательность $\left\{x_{n}\right\}$, полученная методом (5а) или методом (6а), и справедлива оценка

$$
\left\|x^{*}-x_{n}\right\| \leqslant M^{*} \delta_{n} \leqslant \frac{V \overline{2 \beta}}{A} \delta_{n}
$$

где $\left.M^{*}=\lim M_{n} \quad u \quad \delta_{n}=\| P\left(x_{n}\right)\right\rceil$.

Легко убедиться, что для методов (5a) и (6a) имеют место также теоремы, аналогичные теоремам 4,4 а и 5 a.

11. Рассмотренные методы применимы, в частности, и для решения линейного уравнения

$$
P(x) \equiv L x-y=0
$$

где $y-$ заданный элемент пространства $H$. При этом полученные результаты упрощаются, потому что $P^{\prime}(x)=L$ и $P^{\prime \prime}(x)=0$. Так как теперь $\left\|Q^{\prime}(x)\right\|=\|\bar{L} L\|=\|L\|^{2}$, то теорема 1 и 1а (а также теоремы 2 и 2а) совпадают. Мы получим из теорем 1-2а следующие две теоремы:

Те ор ем а 16. Если линейный ограниченный оператор $L$ удовлетворяет условиям:

$$
\begin{aligned}
& 1^{\circ} \quad\|\bar{L} h\| \geqslant \frac{1}{M}\|h\| \text { дляввех } h \in H \quad(M>0), \\
& 2^{\circ} \quad q=\frac{1}{a} \sqrt{\alpha^{2}-2 \alpha+M^{2}\|L\|^{2}}<1,
\end{aligned}
$$

то уравнение $(26)$ имеет в сфере $\left\|x-x_{0}\right\| \leqslant M\left\|P\left(x_{0}\right)\right\|$ решение $x^{*}, \kappa$ которому сходится последовательность $\left\{x_{n}\right\}$, полученная метобом (5) (исходя из произвольно выбранной точки $\left.x_{0}\right)$, и имеет место оценка

$$
\left\|x^{*}-x_{n}\right\| \leqslant \frac{M}{1-q}\left\|P\left(x_{n}\right)\right\| \leqslant \frac{M\left\|P\left(x_{0}\right)\right\|}{1-q} q^{n} .
$$

Т еоре м а 2б. Если линейный ограниченный оператор $L$ удовлетворяет условиям: 
$1^{\circ} \quad|(L h, h)| \geqslant \frac{1}{M}\|h\|^{2}$ для всех $h \in H \quad(M>0)$,

$2^{\circ} \quad q=\frac{1}{a} \sqrt{\alpha^{2}-2 \alpha+M^{2}\|L\|^{2}}<1$,

то уравнение (26) имеет в сфере $\left\|x-x_{0}\right\| \leqslant M\left\|P\left(x_{0}\right)\right\|$ решение $x^{*}, \kappa$ которому сходится последовательность $\left\{x_{n}\right\}$, полученная методом (5) или методом (6) (исходя из произвольно выбранной точки $x_{0}$ ), и имеет место оценка

$$
\left\|x^{*}-x_{n}\right\| \leqslant M\left\|P\left(x_{n}\right)\right\| \leqslant M\left\|P\left(x_{0}\right)\right\| q^{n} .
$$

В случае линейного уравнения остальные теоремы не дают ничего нового по сравнению с теоремами $1-2 \mathrm{a}$, но результаты методов (5a) и (6а), упомянутые в пункте 9, остаются в силе.

Если $\alpha=2$, то теоремы 16 и 26 дают соответствующие теоремы из $\left[{ }^{3}\right]$ и $\left[{ }^{4}\right]$.

\section{Л И Т Е Р А Т У Р А}

1. M. A $1 \mathrm{tm}$ an, Connection Between the Method of Steepest Descent and Newton's Method, Bull. Acad. Polon. Sci., Cl. III, 5, No. 11, 1957, 1031-1036.

2. M. A $1 \mathrm{tman}$, Concerning Approximate Solutions of Non-Linear Functional Equations, Bull. Acad. Polon. Sci., Cl. III, 5, No. 5, 1957, 461-465.

3. M. A $1 \mathrm{tm}$ a n, On the Approximate Solutions of Operator Equations in Hilbert Space, Bull. Acad. Polon. Sci., Cl. III, 5, No. 6, 1957, 605-609.

4. M. A $1 \mathrm{tm}$ a n, Concerning the Approximate Solutions of Operator Equations in Hilbert Space, Bull. Acad. Polon. Sci., Cl. III, 5, No. 7, 1957, 711-715.

5. M. A $1 \mathrm{tman}$, A Note of Approximate Solutions of Non-Linear Operator Equations in Hilbert Space, Bull. Acad. Polon. Sci., Cl. III, 5, No. 8, 1957, 783-787.

6. M. A $1 \mathrm{tm}$ a n, On the Approximate Solutions of Non-Linear Functional Equations, Bull. Acad. Polon. Sci., Cl. III, 5, No. 5, 1957, 457-460.

7. Л. К и в и стик, О методе наискорейшего спуска для решения нелинейных уравнений, Изв, АН ЭССР. Серия физ.-мат. и техн. наук, т. IX, № 2, 1960, 145-159.

Институт энергетики

Академии наук Эстонской ССР
Поступила в редакцию

2. XI 1959

\section{MÕNEDEST ITERATSIOONIMEETODITEST OPERAATORVORRANDITE LAHENDAMISEKS HILBERTI RUUMIS}

\section{Kivistik}

\section{Resümee}

Artiklis käsitletakse operaatorvōrrandi $P(x)=0$ ligikaudset lahendamist iteratsioonimeetoditega (5), (6), (5a), (6a) ja antakse nende meetodite koonduvuseks piisavad tingimused. Erijuhuna vaadeldakse lineaarset võrrandit (punkt 11), mille puhul üldised tulemused lihtsustuvad. 


\section{ON SOME ITERATIVE METHODS FOR SOLVING OPERATOR EQUATIONS IN HILBERT SPACE}

\section{Kivistik}

\section{Summary}

Let $P(x)=0$ be a linear or non-linear operator equation in Hilbert space. For solving this equation the present paper considers the following methods

$$
x_{n+1}=x_{n}-\frac{\left\|P\left(x_{n}\right)\right\|^{2}}{\alpha_{n}\left(P^{\prime}\left(x_{n}\right) P\left(x_{n}\right), P\left(x_{n}\right)\right)} P\left(x_{n}\right)
$$

and

$$
x_{n+1}=x_{n}-\frac{\left\|P\left(x_{n}\right)\right\|^{2}}{\alpha_{n}\left\|Q\left(x_{n}\right)\right\|^{2}} Q\left(x_{n}\right) \text {, }
$$

where $Q(x)=\overline{P^{\prime}(x)} P(x)$ (the linear operator $\overline{P^{\prime}(x)}$ is adjoint of $\left.P^{\prime}(x)\right)$ and $\alpha_{n}(n=$ $=0,1, \ldots)$ are real numbers such that $\frac{1}{2}<\alpha_{n}<\infty$.

The convergence of these methods is proved under several assumptions. The particular case $\alpha_{n}=\alpha(n=0,1, \ldots)$ is especially considered.

Academy of Sciences of the Estonian S.S.R., Institute of Energetics

Received

Nov. 2nd, 1959 